\title{
Foliations and Chern-Heinz inequalities
}

\author{
J. L. M. BARBOSA G. P. BESSA J. F. MONTENEGRO
}

July 28, 2018

\begin{abstract}
We extend the Chern-Heinz inequalities about mean curvature and scalar curvature of graphs of $C^{2}$-functions to leaves of transversally oriented codimension one $C^{2}$-foliations of Riemannian manifolds. That extends partially Salavessa's work on mean curvature of graphs and generalize results of Barbosa-Kenmotsu-Oshikiri [3] and Barbosa-GomesSilveira 2] about foliations of 3-dimensional Riemannian manifolds by constant mean curvature surfaces. These Chern-Heinz inequalities for foliations can be applied to prove Haymann-Makai-Osserman inequality (lower bounds of the fundamental tones of bounded open subsets $\Omega \subset \mathbb{R}^{2}$ in terms of its inradius) for embedded tubular neighborhoods of simple curves of $\mathbb{R}^{n}$.
\end{abstract}

Mathematics Subject Classification (2000): 58C40, 53C42

Key words: Elliptic operator in divergence form, fundamental tone, eigenvalue estimates, $L_{r}$ operator, Newton transformations.

\section{Introduction}

Heinz [14, proved that if the graph of a $C^{2}$-function $z=z(x, y)$ defined on $x^{2}+y^{2}<r^{2}$ has mean curvature $H(x, y)$ satisfying $|H(x, y)| \geq c>0$ then $r \cdot c \leq 1$ and if the Gaussian curvature of the graph satisfies $K(x, y) \geq c>0$ then $r \cdot c^{1 / 2} \leq 1$. As observed by Heinz, these inequalities are a qualitative improvement of inequalities of the form $r \cdot \varrho(c) \leq 1$, for some constant $\varrho(c)$ depending on $c$, implicit in Bernstein's work [4, 5]. Chern [10], extended Heinz inequalities to graphs of $C^{2}$-functions $z=z(x)$ defined on bounded domains $\Omega \subset \mathbb{R}^{n}$ with smooth boundaries $\partial \Omega$, showing that if the mean curvature $H(x)$ of the graphs satisfy $|H(x)| \geq c>0$ then $c \leq \operatorname{vol}_{n-1}(\partial \Omega) / \operatorname{vol}_{n}(\Omega)$ and if the scalar curvatures satisfy $S(x) \geq c>0$ then $c^{1 / 2} \leq \operatorname{vol}_{n-1}(\partial \Omega) / \operatorname{vol}_{n}(\Omega)$. These inequalities are known nowadays as the Chern-Heinz inequalities.

An immediate corollary of the Chern-Heinz inequalities is that an entire graph of a $C^{2}$ function $f: \mathbb{R}^{n} \rightarrow \mathbb{R}$ has constant mean curvature $H$ if and only if $H=0$ or constant nonnegative scalar curvature $S \geq 0$ if and only if $S=0$. 
Salavessa in [18] considered graphs $G(f) \subset M \times N$ of a smooth maps $f: M \rightarrow N$ and proved that if the graph $G(f)$ has parallel mean curvature vector $H_{G(f)}$ then for every oriented compact domain $\Omega \subset M$ one has that $\left\|H_{G(f)}\right\| \leq \operatorname{vol}_{n-1}(\partial \Omega) / \operatorname{vol}_{n}(\Omega)$. Recall that the Cheeger constant of $M$ is defined by $h(M)=\inf _{\Omega}\left(\operatorname{vol}_{n-1}(\partial \Omega) / \operatorname{vol}_{n}(\Omega)\right)$, where the infimum is taken over all relatively compact subsets $\Omega$ of $M$ with smooth boundary. In particular, if $h(M)=0$ then $G(f)$ is minimal. If $\operatorname{dim}(N)=1$ this result is valid regardless the parallelism of its mean curvature vector.

A graph of a smooth function $f: \Omega \subset M \rightarrow \mathbb{R}$ generates by vertical translation a transversally oriented codimension one smooth foliation of $\Omega \times \mathbb{R}$, where the leaves are all isometric to the graph. In this note we show that the Chern-Heinz inequalities can be extended to leaves of transversally oriented codimension-one $C^{2}$-foliations of Riemannian manifolds. Before we state our main result let us recall that the fundamental tone $\lambda^{*}(\Omega)$ of an open subset $\Omega \subset M$ of a smooth Riemannian manifold is defined by

$$
\lambda^{*}(\Omega)=\inf \left\{\frac{\int_{\Omega}|\nabla f|^{2}}{\int_{\Omega} f^{2}}, f \in H_{0}^{1}(\Omega) \backslash\{0\}\right\},
$$

where $H_{0}^{1}(\Omega)$ is the completion of $C_{0}^{\infty}(\Omega)$ w.r.t. the norm $\|\varphi\|_{\Omega}^{2}=\int_{\Omega} \varphi^{2}+\int_{\Omega}|\nabla \varphi|^{2}$. When $\Omega$ is a bounded with piecewise smooth boundary $\partial \Omega$ then $\lambda^{*}(\Omega)$ coincides with the first eigenvalue $\lambda_{1}(\Omega)$ of the Laplacian of $\Omega$.

Theorem 1.1 Let $\mathcal{F}$ be a transversely orientable codimension-one $C^{2}$-foliation of a connected open set $\Omega$ of a Riemannian manifold $M$. Let $\eta$ be a unit vector field on $\Omega$ normal to the leaves of $\mathcal{F}$. Then

$$
2 \sqrt{\lambda^{*}(\Omega)} \geq \inf _{F \in \mathcal{F}} \inf _{x \in F}\left|H_{F}\right|(x)
$$

This theorem has a number of interesting consequences, stated below as corollaries. It imposes strong restrictions for the existence of foliations by constant mean curvature hypersurfaces on open sets with zero fundamental tone or with Ricci curvature bounded below, corollaries 1.2. 1.4. On the other hand if an open set can be foliated by constant mean curvature smooth hypersurfaces then inequality 1.2 gives a lower bound for the fundamental tone, see Theorem (1.9).

Barbosa, Kenmotsu and Oshikiri [3], have studied $C^{3}$-foliations of complete manifolds $M$ whose leaves have constant mean curvature. They have shown that when $M$ is flat, noncompact and all leaves of the foliation have the same mean curvature then leaves are minimal. The following corollary extends their result to Riemannian manifolds with $\lambda^{*}(M)=0$.

Corollary 1.2 Let $\mathcal{F}$ be transversely oriented codimension-one $C^{2}$-foliation of a Riemannian manifold $M$ with $\lambda^{*}(M)=0$. If the leaves $F \in \mathcal{F}$ have the same constant mean curvature then each leaf is minimal.

Remark 1.3 The class of smooth Riemannian manifolds $M$ with $\lambda^{*}(M)=0$ is quite large. It contains all closed, all complete noncompact with nonnegative Ricci curvature. In fact, by 
Cheng's Comparison Theorem [9] all complete Riemannian manifolds with asymptotically nonnegative Ricci curvature has zero fundamental tone. Recall that $M$ has asymptotically nonnegative Ricci curvature if $\operatorname{Ric}_{M}(x) \geq-\psi\left(\right.$ dist $\left._{M}\left(x_{0}, x\right)\right)$, for a continuous function $\psi:[0, \infty) \rightarrow$ $[0, \infty)$ with $\lim _{t \rightarrow \infty} \psi(t)=0, x_{0} \in M$.

Let $\mathcal{F}$ be a transversely oriented codimension-one $C^{2}$-foliation of an open subset $\Omega$ of the $n$-dimensional complete Riemannian manifold $M$ with Ricci curvature bounded below $\operatorname{Ric}_{M} \geq$ $(n-1) \kappa$ by complete oriented surfaces with the same constant mean curvature $H$. BarbosaKenmotsu-Oshikiri, ([3], Theorem 3.1) showed that if $M$ is compact and $\kappa=0$ then the leaves of $\mathcal{F}$ are totally geodesic and the Ricci curvature of $M$ is zero in the directions normal to the leaves. They also showed that if $M=\mathbb{N}^{n}(\kappa)$, the simply connected space form of constant sectional curvature $\kappa$, if $\kappa \leq 0$ and $H \geq(n-1) \sqrt{-\kappa}$ then $H=(n-1) \sqrt{-\kappa}$ ([3], Theorem 3.8).

As a corollary of Theorem (1.1) we extend Theorem 3.8 of Barbosa-Kenmotsu-Oshikiri to $n$-dimensional Riemannian manifolds Ricci curvature bounded below $\operatorname{Ric}_{\mathrm{M}} \geq(n-1) k$.

Corollary 1.4 Let $\mathcal{F}$ be transversely oriented codimension-one $C^{2}$-foliation of a complete $n$ dimensional Riemannian manifold $M$ with Ricci curvature $\operatorname{Ric}_{\mathrm{M}} \geq(n-1) k$. Then

i) $2 \sqrt{\lambda^{*}\left(\mathbb{N}^{n}(k)\right)} \geq \inf _{F \in \mathcal{F}} \inf _{x \in F}\left|H_{F}\right|(x)$.

ii) If $\left|H_{F}\right| \geq c>0$ then $k=-a^{2}$ for some positive a satisfying $(n-1) \cdot a \geq c$.

We observe here that the proof of Theorem 1.5 of [6] can be straight forward adapted to give a version Theorem 3.1 of Barbosa-Kenmotsu-Oshikiri [3] for complete Riemannian manifolds $M$ nonnegative Ricci curvature. We have the following theorem.

Theorem 1.5 Let $\mathcal{F}$ be transversely oriented codimension-one $C^{2}$-foliation of a complete Riemannian manifold $M$ with nonnegative Ricci curvature Ric $_{M} \geq 0$. Suppose that the leaves are complete oriented hypersurfaces with the same constant mean curvature $H$. Then $H=0$ and each leaf $F \in \mathcal{F}$ is stable. If a leaf $F$ is compact then $F$ is totally geodesic and the Ricci curvature of $M$ is zero in the normal directions to $F$.

Remark 1.6 For $n=3$, Schoen [19] showed that a complete stable minimal surface in a 3dimensional Riemannian manifold with nonnegative Ricci curvature is totally geodesic. Thus for $n=3$, Theorem 1.5 fully generalizes Theorem 3.1 of [3].

The graphs $G(f)$ of smooth functions $f: \Omega \rightarrow \mathbb{R}$ generates, by vertical translations, transversally oriented smooth foliations of $\Omega \times \mathbb{R}$. Applying Theorem (1.1) to such foliations we have the following generalizations of Chern-Heinz results. The Corollary (1.7) were proved before by Isabel Salavessa, [18. We state it here because the proof we provide is simpler than hers. 
Corollary 1.7 (Salavessa) Let $f: \Omega \subset M \rightarrow \mathbb{R}$ be a $C^{2}$-function defined on a domain $\Omega$. Consider the product metric on $M \times \mathbb{R}$. Then

$$
\sqrt{\inf |H|} \leq 2 \sqrt{\lambda^{*}(\Omega)}
$$

In particular,

a) if $\Omega=M, \lambda^{*}(M)=0$ and $G(f)$ has constant mean curvature then the graph is a minimal hypersurface.

b) if $\Omega=M, M$ closed and $G(f)$ has constant mean curvature $H$ then $H=0, f$ is constant and the graph is an slice of the product $\Omega \times \mathbb{R}$.

Corollary (1.7) has the following version for scalar curvature.

Corollary 1.8 Let $M$ be an n-dimensional Riemannian manifold with non-positive sectional curvature and $f: \Omega \subset M \rightarrow \mathbb{R}$ be a $C^{2}$-function defined on a domain $\Omega$. Consider the product metric on $M \times \mathbb{R}$ and suppose that the scalar curvature $S$ of the graph $G(f) \subset M \times \mathbb{R}$ satisfies $S \geq 0$. Then

$$
\sqrt{\inf S} \leq 2 \sqrt{\lambda^{*}(\Omega)}
$$

In particular, if $\Omega=M, \lambda^{*}(M)=0$ and the graph $G(f)$ has constant nonnegative scalar curvature $S \geq 0$ then $S=0$.

Our last result is an extension of the Haymann-Makai-Osserman inequality to tubular neighborhood of simple curves of $\mathbb{R}^{n}$. Recall that the inradius $\rho(\Omega)$ of a connected open set $\Omega \subset M$ of a Riemannian manifold is defined as $\rho(\Omega)=\sup \left\{r>0 ; B_{M}(r) \subset \Omega\right\}$, where $B_{M}(r)$ is a ball of radius $r$ of $M$. In [15], Makai proved that the fundamental tone $\lambda^{*}(\Omega)$ of a simply connected bounded domain $\Omega \subset \mathbb{R}^{2}$ with smooth boundary and inradius $\rho=\rho(\Omega)$ was bounded below by $\lambda_{1}(\Omega) \geq 1 / 4 \rho^{2}$. Unaware of Makai's result, Haymann [13] proved years later that $\lambda_{1}(\Omega) \geq 1 / 900 \rho^{2}$. Osserman [16] among other things improved Haymann's estimate back to $\lambda_{1}(\Omega) \geq 1 / 4 \rho^{2}$. The Haymann-Makai-Osserman inequality is only known to be valid for simply connected bounded open sets $\Omega \subset \mathbb{R}^{2}$ with smooth boundary. Theorem (1.1) can be seen as (a weak form of) Haymann-Makai-Osserman inequality in higher dimension. For certain foliated open sets of $\mathbb{R}^{n}$ like embedded tubular neighborhood of simple smooth curves Theorem (1.1) can be stated as follows.

Theorem 1.9 Let $\gamma: I \subset \mathbb{R} \rightarrow \mathbb{R}^{n}$ be a simple smooth curve. Let $T_{\gamma}(\rho)$ be an embedded tubular neighborhood of $\gamma$ with variable radius. Let $\rho>0$ be its inradius. Then

$$
\lambda^{*}\left(T_{\gamma}(\rho)\right) \geq \frac{(n-1)^{2}}{4 \rho^{2}}
$$




\section{Foliations and Chern-Heinz Inequalities}

\subsection{Proof of Theorem (1.1)}

Let $\mathcal{F}$ be a transversally oriented codimension one $C^{2}$-foliation of a connected open subset $\Omega$ of a Riemannian manifold $M$. This means that there is a unit vector field $\eta$ on $\Omega$ normal to the leaves. We may suppose that the mean curvature $H_{F}$ of the leaves $F \in \mathcal{F}$ are such that $\inf _{F \in \mathcal{F}} \inf _{x \in F}\left|H_{F}(x)\right|>0$ otherwise there is nothing to prove. That means that the mean curvatures of the leaves $H_{F},\left(\vec{H}_{F}=H_{F} \cdot \eta\right)$, does not change sign in $\Omega$. Thus we may suppose that the mean curvature $H_{F}$ is positive and bounded from zero, that is, there is a positive constant $c_{F}$ such that the mean curvature $H_{F}$ computed with respect to the vector field $\eta$ satisfies $H_{F} \geq c_{F}$. The divergence $\operatorname{div} \eta$ at a point $x \in F$ is given by $\operatorname{div} \eta(x)=H_{F}(x)$. On the other hand Bessa and Montenegro 7 proved that the fundamental tone of $\Omega$ is bounded below by $\lambda^{*}(\Omega) \geq c(\Omega)^{2} / 4$ where $c(\Omega)=\sup _{X}\left(\inf _{\Omega} \operatorname{div} X /\|X\|_{\infty}\right)$ with the supremum taken over all smooth vector fields $X$ on $\Omega$ such that $\inf _{\Omega} \operatorname{div} X>0$ and $\|X\|_{\infty}=\sup _{\Omega}|X|<\infty$. The estimates for the fundamental tone implies that

$$
\lambda^{*}(\Omega) \geq\left[\inf _{F \in \mathcal{F}} \inf _{x \in F} H_{F}(x)\right]^{2} / 4
$$

This is equivalent to (2).

\subsection{Proof of the corollaries}

The Corollary (1.2) follows immediately from Theorem (1.1). The Corollary (1.4) is a direct consequence of Theorem (1.1) and the Cheng's Comparison Theorem. For if $B_{M}(r)$ is a geodesic ball of radius $r$ in an $n$-dimensional Riemannian manifold $M$ with Ricci curvature $\operatorname{Ric}_{M} \geq$ $(n-1) k$ bounded below, Cheng 's Comparison Theorem says that $\lambda^{*}\left(B_{M}(r)\right) \leq \lambda^{*}\left(B_{\mathbb{M}(k)}(r)\right)$, where $B_{\mathbb{M}(k)}(r)$ is a geodesic ball of radius $r$ in the simply connected $n$-dimensional space $\mathbb{M}(k)$ of constant sectional curvature $k$. In particular $\lambda^{*}(M) \leq \lambda^{*}(\mathbb{M}(k))$. Thus, by inequality 2 we have that

$$
\inf _{F \in \mathcal{F}} \inf _{x \in F}\left|H_{F}\right|(x) \leq 2 \sqrt{\lambda^{*}(M)} \leq 2 \sqrt{\lambda^{*}(\mathbb{M}(k))}
$$

If $\left|H_{F}\right| \geq c>0$ we have that $\sqrt{\lambda^{*}(\mathbb{M}(k))} \geq c / 2$. But $\lambda^{*}(\mathbb{M}(k)) \neq 0$ if and only if $k=-a^{2}$ in which case $\lambda^{*}(\mathbb{M}(k))=(n-1)^{2} a^{2} / 4$ and $(n-1) a \geq c$.

\subsection{Proof of Theorem 1.5}

We reproduce here the proof of Theorem 1.5 of [6] with proper modifications that yields the proof of Theorem 1.5. Let $\mathcal{F}$ be transversely oriented codimension-one $C^{2}$-foliation of a complete Riemannian manifold $M$ with bounded geometry and nonnegative Ricci curvature $\operatorname{Ric}_{M} \geq 0$. By hypothesis the leaves are complete oriented hypersurfaces with the same constant mean curvature $H$. By inequality 2 each leaf $F$ is minimal. For each point $p$ of $M$ there is an 
oriented leaf $p \in F_{p} \in \mathcal{F}$. Let $\nu$ be a continuous unit vector field normal to $F_{p}$. There is a sequence of leaves $F_{i}$ of the foliation that converges to $F_{p}$ by the side $\nu$ is pointing. Let $L=\triangle+\operatorname{Ric}(\nu)+|A|^{2}$ the Jacobi operator on $F_{p}$, where $\triangle$ is the Laplacian on $F_{p}, \operatorname{Ric}(\nu)$ is the Ricci curvature of $M$ in the direction $\nu$ and $|A|$ is the norm of the second fundamental form of $F_{p} \subset M$. Take a compact subsets $C \subset C^{\prime} \subset F_{p}$ and consider a sequence of compacts $C_{i} \subset F_{i}$ converging to $C^{\prime}$. We may assume that the only solution of $L(v)=0$ in $C^{\prime}$ with $v=0$ on $\partial C^{\prime}$ is $v \equiv 0$. Thus there exists a function $u \in C^{\infty}\left(C^{\prime}\right)$ with $L(u)=1$ in $C^{\prime}$ and $u=0$ on $\partial C^{\prime}$. The mean curvature $H(t),|t|<\epsilon$ of the immersions $\psi_{t}: C^{\prime} \rightarrow M$ given by $\psi_{t}(x)=\exp _{x}(t u(x) \nu(x))$ has derivative at $t=0$ given by $2 H^{\prime}(0)=L(u)=1$ on $C^{\prime}$, see [17] Thus for small $0<t<\epsilon$, $H(t)>0$. If $u$ is positive at some interior point of $C$ then $\psi_{t}\left(C^{\prime}\right)$ has a tangency point with some $C_{i}$ and this is not possible by the maximum principle. Thus $u \leq 0$. If $u(q)=0$ at an interior point of $C^{\prime}$ we have that $u \equiv 0$ and this is impossible. Thus $u<0$ in the interior of $C^{\prime}$ and $u=0$ on $\partial C^{\prime}$. Setting $w=-u$ we have that $w>0$ in the interior of $C^{\prime}$ with $L(w) \leq 0$. In particular $w$ restricted to $C$ is a positive function. Let $u_{1}$ be the first eigenfunction of $C$, i.e $L\left(u_{1}\right)+\lambda_{1}(C) u_{1}=0$. Suppose by contradiction that $\lambda_{1}(C)<0$. Let $h=w-t u_{1}>0$ for small $t$. We have that $L(h)=L(w)-t L\left(u_{1}\right) \leq t \lambda_{1}(C) u_{1}<0$ on $C$. Then $\triangle h<0$ and $h$ has a minimum in the interior of $C$. By the maximum principle $h$ is constant. Choosing $t$ in such way that this minimum is zero we have a contradiction. Thus $\lambda_{1}(C) \geq 0$ and $C$ is stable. If $F_{p}$ is not compact we can exhaust $F_{p}$ by stable compact sets and $F_{p}$ is stable. If $F_{p}$ is compact, by a result of Fisher-Colbrie [1] there is a positive function $g: F_{p} \rightarrow \mathbb{R}$ solution to $\triangle g-q g=0,-q=\operatorname{Ric}(\nu)+|A|^{2}$. Integrating over $F_{p}$ we have that $\int_{F_{p}} \triangle g-q g=\int_{F_{p}}-q g=0$. This implies that $q=0$ thus $\operatorname{Ric}(\nu)=0$ and $|A|^{2}=0$. The stability operator in $F_{p}$ is then $L=\triangle$. If $F_{p}$ is not stable then there exists a function $f: F_{p} \rightarrow \mathbb{R}$ with $\int_{F_{p}} f=0$ satisfying $\triangle f+\lambda_{1}\left(F_{p}\right) f=0, \lambda_{1}\left(F_{p}\right)<0$. Let $D_{f}=\left\{x \in F_{p}, f(x)>0\right\}$ be a nodal set of $f$. Thus $\lambda_{1}\left(D_{f}\right) \leq \int_{F_{p}} f \triangle f / \int_{F_{p}}<0$. This contradicts the fact that $\lambda_{1}(C)>0$ proved before.

\subsection{Graphs}

The proof of item i) Corollary (1.7) are immediate once we have inequality 2, Since a graph generates an oriented codimension one $C^{2}$-foliation of $\Omega \times \mathbb{R}$. The item ii) it is also straight forward since $M$ is closed $\lambda^{*}(M)=0$. From inequality 2 we have that $H=0$. Therefore $G(f)$ besides being compact it is minimal. Observe that the slices $M \times\{t\}$ are totally geodesic in $M \times \mathbb{R}$. By the maximum principle the leaves are $M \times\{t\}$. The proof of Corollary 1.8 is as follows. We may suppose that $c=\inf S>0$ otherwise there is nothing to prove. Let $p \in G(f)$ and $\left\{e_{1}, \cdots e_{n}\right\}$ be an orthonormal basis for the tangent space $T_{p} G(f)$ of the graph $G(f) \subset M \times \mathbb{R}$. The Gauss equation for the plane generated by $e_{i}, e_{j}$ is:

$$
K\left(e_{i}, e_{j}\right)=\bar{K}\left(e_{i}, e_{j}\right)+\left\langle\mathrm{A}\left(e_{i}, e_{i}\right), \mathrm{A}\left(e_{j}, e_{j}\right)\right\rangle-\left|\mathrm{A}\left(e_{i}, e_{j}\right)\right|^{2}
$$

where $K$ represents the Gaussian curvature of $G(f), \bar{K}$ the sectional curvature of the ambient space $\Omega \times \mathbb{R}$ and $\mathrm{A}$ the second fundamental form of the graph. Adding these equations

$$
S(p)=\sum_{i, j} \bar{K}\left(e_{i}, e_{j}\right)+H^{2}-\|\mathrm{A}\|^{2}
$$


Since the sectional curvatures of $M$ are non-positive one has then $\bar{K} \leq 0$. It follows that $S(p) \leq H^{2}(p)$. Now, if $S \geq c>0$ then $H \geq \sqrt{c}>0$ and by (3) we have (41).

\subsection{Haymann-Makai-Osserman inequality}

Let $\gamma: I \rightarrow \mathbb{R}^{n}$ be a simple curve and $T_{\gamma}(\rho)$ be an embedded tubular neighborhood of $\gamma$ with inradius $\rho$. Recall that the inradius of an open connected set $\Omega$ is defined as $\sup \{r>$ $0, B(r) \subset \Omega\}$. The Haymann-Makai-Osserman inequality says that the fundamental tone of an open connected, simply connected, subsets of $\mathbb{R}^{2}$ with smooth boundary has a lower bounded depending only on the inradius, $\lambda^{*}(\Omega) \geq 1 /(2 \rho)^{2}, \rho=\rho(\Omega)$ the inradius of $\Omega$. We have a weak version of this inequality in higher dimension, since we have it only for embedded tubular neighborhoods. However, these tubes can knotted and the curve can be closed. The idea of the proof is trivial application of inequality 2. An embedded tube $T_{\gamma}(\rho)$ with inradius can be foliated by spherical caps of radius $\rho$. Just run with the center of a ball of radius $\rho$ along $\gamma$. This gives a foliation whose leaves have constant mean curvature $(n-1) / \rho$. Thus $\lambda^{*}\left(T_{\gamma}(\rho)\right) \geq(n-1)^{2} / 4 \rho^{2}$.

Acknowledgments: The research and writing of this work was partially supported by CNPqBrazil.

\section{References}

[1] H. Alencar, M. do Carmo. Hypersurfaces of constant mean curvature with finite index and volume of polynomial growth. Arch. Math. 60 (1993), 489-493.

[2] J. L. M. Barbosa, J. M. Gomes, A. M. Silveira. Foliations of 3-dimensional space forms by surfaces with constant mean curvature Bol. Soc. Bras. Mat. 18 (1987), 1-12.

[3] J. L. M. Barbosa K. Kenmotsu, O. Oshikiri. Foliations by hypersurfaces with constant mean curvature. Math. Z. 207 (1991), 97-108.

[4] S. Bernstein. Sur la généralisation du problème de Dirichlet. Math. Ann. 69 (1910), 82-136.

[5] S. Bernstein. Sur les surfaces définies au moyen de leur courbure moyenne ou totale. Ann. Ecole. Norm. Sup. 27 (1909), 233-256.

[6] G. P. Bessa L. P. Jorge, G. Oliveira-Filho. Half-space theorems for minimal surfaces. J. Differenctial Geom. 57 (2001) 493-508.

[7] G. P. Bessa, J. F. Montenegro. Eigenvalue estimates for submanifolds with locally bounded mean curvature Ann. Global Anal. and Geom. 24 (2003), 279-290.

[8] J. Cheeger. A lower bound for the smallest eigenvalue of the Laplacian. Problem in Analyis Princenton Univ. Press, New Jersey, (1970), 195-199. 
[9] S. Y. Cheng. Eigenvalue comparison theorems and its geometric applications. Math. Z. 143 (1975), 289-297.

[10] S. S. Chern. On the curvature of a piece of hypersurface in Euclidean space. Abh. Math. Sem Hamburg 29 (1965), 77-91.

[11] D. Fischer-Colbrie R. Schoen. The structure of complete stable minimal surfaces in 3manifolds of nonnegative scalar curvature. Comm. Pure. Appl. Math. 33 (1980), 199-211.

[12] M. F. Elbert. Constant positive 2-mean curvature hypersurfaces. Illinois J. Math. 46 (2002), 247-267.

[13] W. Haymann. Some bounds for principal frequency. Applicable Anal. 7 (1978), 247-254.

[14] E. Heinz. Über Flächen mit eindeutiger projektion auf eine ebene, deren krümmungen durc ungleichungen eingschränkt sind. Math. Ann. 129 (1955), 451-454.

[15] E. Makai. A lower estimation of the principal frequencies of simply connected membranes. Acta Math. Acad. Sci. Hungar. 16 (1965), 319-366.

[16] R. Osserman. A note on Hymann's theorem on the bass note of a drum. Comment. Math. Helv. 52 (1977), 545-555.

[17] Compactness of spaces of properly embedded minimal surfaces with finite total curvature. Indiana Univ. Math. J 44 (1995), 139-152.

[18] Isabel M. C. Salavessa. Graphs with parallel mean curvature. Proc. Amer. Math. Soc. 107 (1989), 449-458.

[19] R. Schoen. Estimates for stable minimal surfaces in three dimensional manifolds. Ann. of Math. Stud. 103 (1983) 111-126. 